\section{CRAF, acteur-clé dans les adénocarcinomes pulmonaires induits par l'oncogène K-Ras}

Sarah Francoz, Pierre Dubus
Experimental Oncology Group

CNIO-Centro Nacional de Investigaciones Oncológicas (Spanish National Cancer Research Centre), Melchor Fernández Almagro, 3, 28029 Madrid, Espagne.

\section{sefrancoz@cnio.es}

EA2406, Histologie et pathologie moléculaire des tumeurs, Université de Bordeaux,

146, rue Léo Saignat, 33076 Bordeaux, France ;

Laboratoire de biologie des tumeurs, hôpital du Haut Lévêque, CHU de Bordeaux, 33604 Pessac, France.

pierre.dubus@u-bordeaux2.fr Depuis ces 20 dernières années, le cancer du poumon est devenu la première cause de mortalité par cancer dans le monde. Les gènes RAS sont mutés dans $50 \%$ des adénocarcinomes pulmonaires et $20 \%$ à $30 \%$ des carcinomes pulmonaires non à petites cellules (CPNPC), le type de tumeur pulmonaire le plus fréquent chez l'homme. Les protéines RAS sont impliquées dans diverses fonctions cellulaires essentielles pour l'initiation et la progression tumorales, comme la prolifération cellulaire, la différenciation, la mobilité et l'apoptose (pour revue, voir [1]). Jusqu'à présent, les différentes stratégies visant à inhiber pharmacologiquement les oncoprotéines RAS n'ont pas abouti. Une alternative, cibler les effecteurs situés en aval des RAS à des fins thérapeutiques, a été étudiée. Parmi les différentes voies de signalisation activées par les protéines RAS, la voie ERK (extracellular signalregulated kinase)-MAPK (mitogen-activated protein kinase) semble être principalement impliquée dans le contrôle de la prolifération cellulaire, essentielle au développement tumoral [2]. L'analyse de cette voie de signalisation dans le développement des CPNPC apparaît donc particulièrement pertinente.

Après activation de récepteurs par des stimulus extracellulaires, les GTPases RAS se lient au GTP et recrutent les sérine/thréonine kinases RAF à la membrane cytoplasmique (Figure I). Les RAF activées phosphorylent et activent les kinases MEK (mitogen-activated kinase) ERK kinase) qui, à leur tour, activent les transcription impliqués dans le contrôle

kinases ERK. Ces protéines phosphorylent notamment de nombreux facteurs de de la prolifération cellulaire $(\rightarrow)$.

$(\rightarrow)$ Voir l'article de N. Dumaz et al.,

Modèle murin de carcinome pulmonaire non à petites cellules

La majorité des mutations des gènes RAS (90\%) observées dans les CPNPC affectent $K-R A S$, essentiellement au niveau du codon 12. La souris K-Ras ${ }^{+/ L S L G 12 V g e o}$, générée dans le laboratoire de Mariano Barbacid [3], permet d'exprimer I'oncogène $K$-Ras ${ }^{\mathrm{V} 12}$ après activation par la recombinase Cre. Ces animaux développent des adénomes et adénocarcinomes pulmonaires quelques mois après traitement par la Cre, et ont été utilisés comme modèle d'étude des CPNPC (pour revue, voir [4]). Afin de déterminer quelle kinase de la voie de signalisation Raf/Erk/Mek est essentielle pour la signalisation de K-Ras ${ }^{\mathrm{V} 12}$ lors de I'initiation des CPNPC, ces animaux ont été croisés avec des souris dont chacune des kinases Raf, Mek et Erk était inactivée, soit constitutivement, soit de façon conditionnelle [5].

Les kinases $\varepsilon$ rk et Mek sont essentielles au développement des CPNPC induits par K-Ras ${ }^{\mathrm{V} 12}$... mais aussi à la survie des souris !

Les souris K-Ras ${ }^{+/ L S L G 12 V g e o}$ ont été croisées avec les souris $\varepsilon r k 1^{-/-}, \varepsilon_{r k} 2^{\text {lox/lox }}$, Mekl $1^{\text {lox/lox }}$ et $M e k 2^{-/-}$. Afin de cibler spécifiquement le tissu pulmonaire, les souris sont traitées avec un adénovirus (par voie intratrachéale) codant pour la recombinase Cre. Ceci permet d'exprimer page 817 de ce numéro
I'oncoprotéine $\mathrm{K}-\mathrm{Ras}^{\mathrm{V} 12}$ et de bloquer l'expression des allèles floxés ( $E r k 2^{l o x}$ ou Mek $1^{\text {lox }}$ ) simultanément et dans les mêmes cellules. Contrairement à l'ablation d'une seule des deux kinases Mek ou Erk, l'élimination concomitante des deux protéines Mek ou Erk dans le tissu pulmonaire altère significativement le nombre de tumeurs induites par l'oncogène $K$-Ras et conduit à une augmentation de la survie de ces animaux (Figure 2). Les quelques tumeurs présentes conservent au moins un allèle $\varepsilon r k 2^{\text {lox }}$ ou Mek $1^{\text {lox }}$ non recombiné, démontrant qu'elles ne peuvent se développer en l'absence des deux kinases Erk ou Mek. Ces observations démontrent que la voie de signalisation Erk-Mapk est essentielle pour les propriétés oncogéniques de $K$-Ras dans ce modèle murin de CPNPC [5]. Afin d'étudier l'implication des kinases Erk et Mek dans l'homéostasie normale chez l'animal adulte, les souris ${\varepsilon r k 1^{-/-}}_{2}$ Erk2lox/lox ; RERT ert/ert et Mek1 lox/lox $\mathrm{Mek}^{-/-}$; $R E R T^{\text {ert/ert }}$ ont été générées. La lignée $R E R T^{\text {ert/ert }}$ exprime la CreERT2 (Cre recombinase inductible par le tamoxifène) de façon ubiquitaire. L'ablation ubiquitaire des kinases Mek ou Erk chez la souris adulte est létale en quelques semaines (Figure 2). Par ailleurs, les inhibiteurs de MEK Cl-1040, AZD6244 et PD0325901, testés à des doses tolérées dans des essais cliniques de phase précoce, montrent leur absence d'activité antitumorale sur des CPNPC [6-8]. Un autre inhibiteur de 


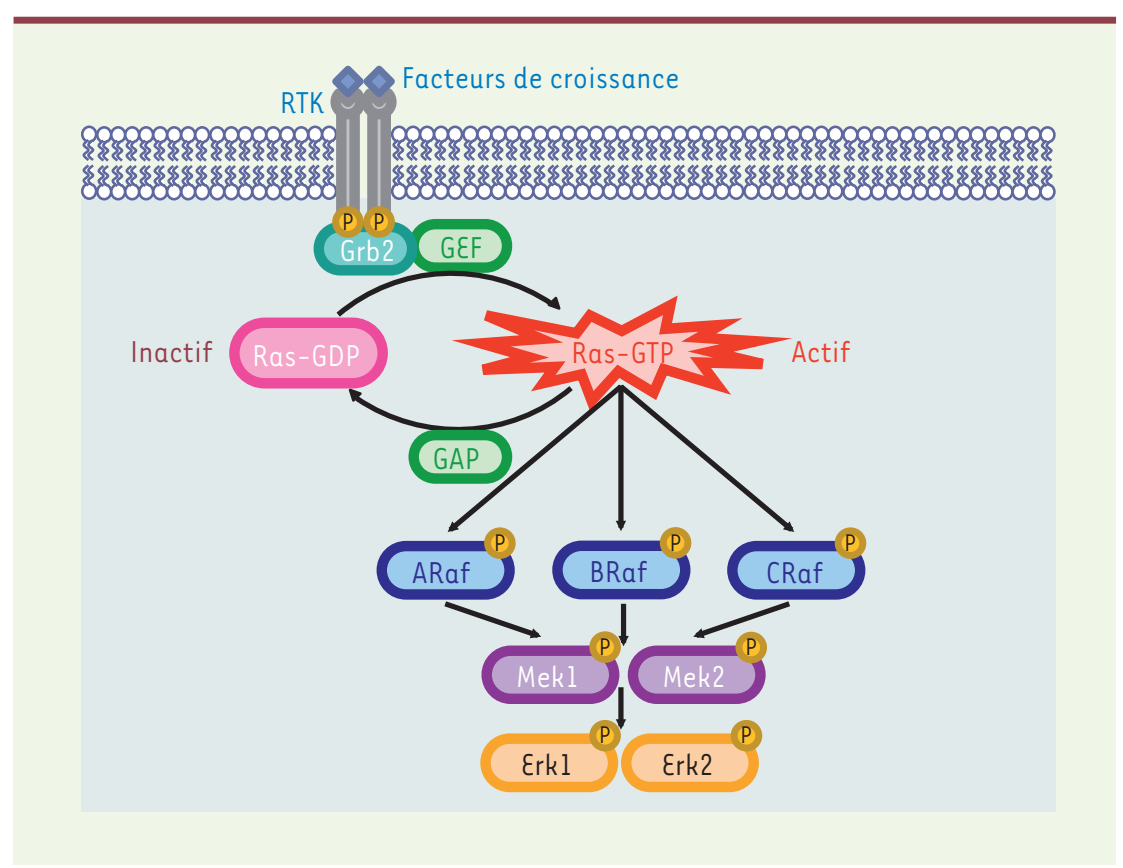

Figure 1. La voie de signalisation Ras/Raf/Mek/Erk. La liaison de facteurs de croissance au récepteur à activité tyrosine kinase (RTK) conduit à l'autophosphorylation du récepteur et au recrutement de la protéine adaptatrice Grb2 (growth factor receptor bound 2) qui se lient également aux G\&F (guanine nucleotide-exchange factors). Grb2-GEF induisent l'activation des GTPases Ras par liaison au GTP. Les Ras-GTP recrutent alors les kinases Raf à la membrane où elles seront activées par phosphorylation. Les Raf phosphorylent ensuite les kinases Mek qui vont à leur tour phosphoryler les kinases Erk. Les protéines GAP (GTPase-activating proteins) permettent l'hydrolyse du GTP en GDP et l'inactivation des Ras.

MEK : GSK1120212, a permis de stabiliser la maladie chez 9 des 14 patients traités atteints de CPNPC mutés pour K-RAS ${ }^{1}$. L'ensemble de ces résultats confirme l'intérêt des inhibiteurs dirigés contre ERK ou MEK dans le traitement des CPNPC mutés pour $K-R A S$, tout en soulignant la difficulté de trouver des doses thérapeutiques efficaces sur la tumeur et tolérées par le patient au niveau systémique.

Analyse de l'implication des protéines Raf dans l'émergence des adénocarcinomes pulmonaires mutés pour K-Ras

\section{Un rôle-clé pour c-Raf}

Les kinases Raf sont les effecteurs directs de K-Ras au sein de la voie Erk-Mapk. De façon surprenante, l'ablation de BRaf n'a pas d'effet sur le développement

\footnotetext{
${ }^{1}$ http://www.esmo.org/events/milan-2010-congress
}

CRAF cible thérapeutique potentielle des CPNPC exprimant l'oncogène K-Ras ${ }^{\mathrm{V} 12}$

Dans un autre modèle, celui des mélanocytes, BRAF active la voie de signalisation ERK-MAPK, alors que dans les mélanomes mutés pour RAS, c'est CRAF qui joue ce rôle [9] $(\rightarrow)$. De plus, un modèle in vivo a permis de démontrer que CRAF est essentiel au développement

$(\rightarrow)$ Voir l'article de Nicolas Dumaz et al., page 817 de ce numéro et au maintien de tumeurs épidermiques induites par Ras [10]. L'ensemble de ces résultats suggère également que CRAF serait impliqué dans le développement de différents types de tumeurs mutées pour RAS.

Les inhibiteurs de RAF possédant une activité dirigée contre BRAF et CRAF inhibent la prolifération des tumeurs mutées pour BRAF ${ }^{V 60 \varepsilon}$, en bloquant la voie ERK$\operatorname{MAPK}[11,12](\rightarrow)$. Au contraire, dans un contexte RAS mutant ou BRAF/RAS sauvage, ces inhibiteurs induisent une augmentation de la croissance tumorale en activant la voie de signalisation ERKMAPK. Par ailleurs, un tiers des patients atteints d'un mélanome et traités avec un inhibiteur de BRAF développent des carcinomes épidermoïdes cutanés [13, 14]. Il reste à déterminer si des inhibiteurs spécifiques de CRAF auraient le même effet.

D'autres modèles ont démontré l'importance de l'inhibition de Cdk4 [15] et NFKB [16] dans le développement des CPNPC ayant une mutation de K-Ras. Ainsi, l'association d'inhibiteurs de CRAF, Cdk4 et NFKB pourrait avoir un effet synergique dans le traitement des tumeurs pulmonaires.

\section{Perspectives}

Le modèle présenté ici a permis d'identifier les gènes de la voie de signalisation Erk-Mapk impliqués dans l'initiation des CPNPC induits par K-Ras ${ }^{\mathrm{V} 12}$. De nouveaux modèles murins, permettant l'étude de l'importance de ces gènes dans la maintenance et la progression des adénocarcinomes pulmonaires mutés pour K-Ras, sont actuellement en cours de mise 
A

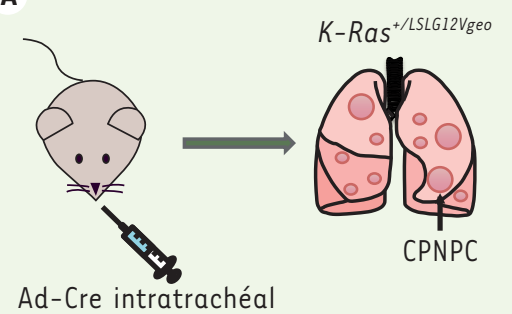

B

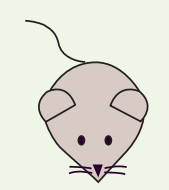

Tamoxifène

$R \varepsilon R T^{\text {ert/ert }}$

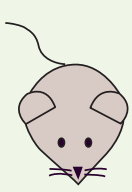

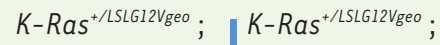
$\varepsilon r k 1^{-/-} ; \varepsilon r k 2^{\text {lox/lox }}$ Mekl lox/lox $;$ Mek2

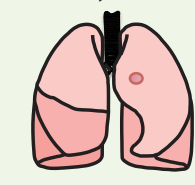

I

I

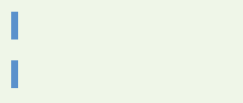

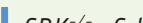

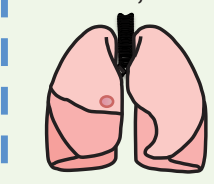

I

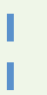

; IMek1 1ox/lox $;$ Mek2 $2^{-/ /}$;

$R \varepsilon R T^{\text {ert/ert }}$

IRERT $T^{\text {ert/ert }}$
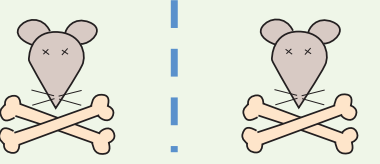

K-Ras ${ }^{+/ L L L G 12 v g e o}$;

$c-R a f^{l o x / l o x}$
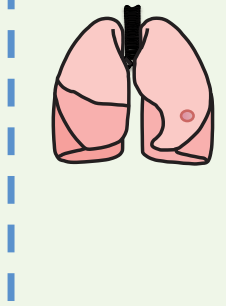

I

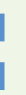

Figure 2. Recherche de cible thérapeutique pour le traitement des CPNPC mutés pour $K$-Ras. $A$. Ablation des gènes de la voie Erk-Mapk lors de l'initiation de tumeur pulmonaires induites par l'oncongène K-Ras ${ }^{\mathrm{V} 12}$. L'ablation concomitante des deux protéines Erk, des deux protéines Mek ou de craf affecte significativement le développement des CPNPC mutés pour K-Ras. B. Ablation systémique des gènes de la voie Erk-Mapk chez la souris adulte. Seule l'ablation systémique de craf permet la survie des animaux.

au point. Ils permettront la séparation temporelle entre l'expression de l'oncogène $K-R_{a s}{ }^{V 12}$ et la délétion des gènes de la voie, une fois que les tumeurs sont établies. Les résultats obtenus à partir de ces nouveaux modèles devraient fournir des informations-clés pour le développement de thérapies ciblées pour le traitement de CPNPC mutés pour K-RAS. $\diamond$

CRAF, a key player in lung adenocarcinomas induced by K-Ras oncogene

\section{CONFLIT D'INTÉRÊTS}

Les auteurs déclarent n'avoir aucun conflit d'intérêts concernant les données publiées dans cet article.

\section{RÉFÉRENCES}

1. Malumbres M, Barbacid M. RAS oncogenes: the first 30 years. Nat Rev Cancer 2003 ; 3 : 459-65.

2. McKay MM, Morrison DK. Integrating signals from RTKs to ERK/MAPK. Oncogene 2007 ; 26 : 3113-21.

3. Guerra C, Mijimolle N, Dhawahir A, et al. Tumor induction by an endogenous K-ras oncogene is highly dependent on cellular context. Cancer Cell $2003 ; 4$ : 111-20.

4. Dubus $P$. CDK4, cible thérapeutique des adénocarcinomes pulmonaires porteurs d'une mutation de l'oncogène KRAS. Med Sci (Paris) 2010 ; $26: 1023-5$.

5. Blasco RB, Francoz S, Santamaria D, et al. c-Raf, but not B-Raf, is essential for development of K-Ras oncogene-driven non-small cell lung carcinoma. Cancer Cell $2011 ; 19: 652-63$.

6. Rinehart J, Adjei AA, Lorusso PM, et al. Multicenter phase II study of the oral MEK inhibitor, $\mathrm{Cl}-1040$, in patients with advanced non-small-cell lung, breast, colon, and pancreatic cancer. J Clin Oncol 2004 ; 22 : 4456-62.

7. Hainsworth JD, Cebotaru CL, Kanarev V, et al. A phase II, open-label, randomized study to assess the efficacy and safety of AZD6244 (ARRY-142886) versus pemetrexed in patients with non-small cell lung cancer who have failed one or two prior chemotherapeutic regimens. J Thorac Oncol 2010 ; 5 : 1630-6.
8. Haura EB, Ricart AD, Larson TG, et al. A phase II study of PD-0325901, an oral MEK inhibitor, in previously treated patients with advanced non-small cell lung cancer. Clin Cancer Res 2010 ; $16: 2450-7$.

9. Dumaz N, Hayward R, Martin J, et al. In melanoma, RAS mutations are accompanied by switching signaling from BRAF to CRAF and disrupted cyclic AMP signaling. Cancer Res 2006 ; 66 : 9483-91.

10. Ehrenreiter K, Kern F, Velamoor V, et al. Raf-1 addiction in ras-induced skin carcinogenesis. Cancer Cell $2009 ; 16: 149-60$.

11. Poulikakos PI, Rosen N. Mutant BRAF melanomasdependence and resistance. Cancer Cell 2011; 19 : 11-5.

12. Hatzivassiliou G, Song K, Yen I, et al. RAF inhibitors prime wild-type RAF to activate the MAPK pathway and enhance growth. Nature $2010 ; 464: 431-5$.

13. Bollag G, Hirth P, Tsai J, et al. Clinical efficacy of a RAF inhibitor needs broad target blockade in BRAFmutant melanoma. Nature $2010 ; 467$ : 596-9.

14. Flaherty KT, Puzanov I, Kim KB, et al. Inhibition of mutated, activated BRAF in metastatic melanoma. N Engl J Med 2010 ; 363 : 809-19.

15. Puyol M, Martin A, Dubus P, et al. A synthetic lethal interaction between K-Ras oncogenes and $\mathrm{Cdk} 4$ unveils a therapeutic strategy for non-small cell lung carcinoma. Cancer Cell $2010 ; 18: 63-73$.

16. Meylan $\varepsilon$, Dooley AL, Feldser DM, et al. Requirement for NF-kappaB signalling in a mouse model of lung adenocarcinoma. Nature 2009 ; 462 : 104-7.

Tarifs d'abonnement M/S - 2011

$>$ Grâce à $\mathrm{m} / \mathrm{s}$, vivez en direct les progrès des sciences biologiques et médicales

Abonnez-vous

à Médecine/Sciences

Bulletin d'abonnement page 798 dans ce numéro de $\mathrm{m} / \mathrm{s}$
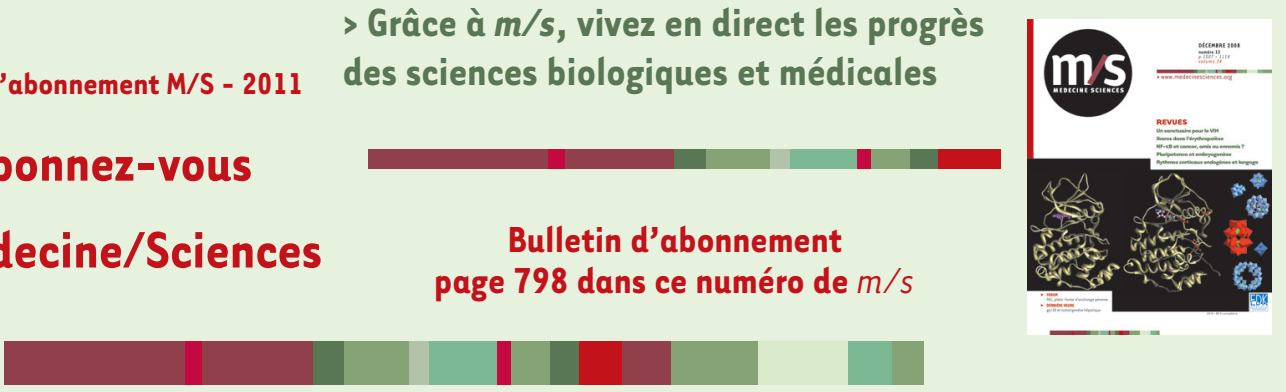